\title{
An Unusual Case of Anterior Vaginal Wall Cyst
}

\author{
${ }^{1} \mathrm{GS}$ Anitha, ${ }^{2} \mathrm{M}$ Prathiba, ${ }^{3}$ Mangala Gowri
}

\begin{abstract}
Vaginal cysts are rare and are mostly detected as an incidental finding during a gynecological examination. Gartner duct cysts, the most common benign cystic lesion of the vagina, represent embryologic remnants of the caudal end of the mesonephric (Wolffian) duct. These cysts are usually small and asymptomatic and have been reported to occur in as many as $1 \%$ of all women. A 17-year-old unmarried girl presented with mass per vagina since one and a half year. On examination, anterior vaginal wall cyst of $8 \times 4 \times 3 \mathrm{~cm}$ was detected. Surgical excision of the cyst was done under spinal anesthesia by sharp and blunt dissection. The cyst was filled with mucoid material and histopathological examination confirmed Gartner origin. This is a rare case of large Gartner cyst.
\end{abstract}

Keywords: Anterior vaginal wall cyst, Gartner cyst, Müllerian cyst, Surgical excision.

How to cite this article: Anitha GS, Prathiba M, Gowri M. An Unusual Case of Anterior Vaginal Wall Cyst. J South Asian Feder Obst Gynae 2015;7(3):227-230.

Source of support: Nil

Conflict of interest: None

Date of received: 4 August 2015

Date of acceptance: 24 September 2015

Date of publication: December 2015

\section{INTRODUCTION}

Vaginal cysts are rare and are mostly detected as an incidental finding during a gynecological examination. ${ }^{1}$ The commonest type of simple vaginal cyst is the Müllerian cyst arising from paramesonephric duct remnants. ${ }^{2}$ The vaginal cysts are divided into the following types depending upon the histology of their lining epithelium: Müllerian cysts (30\%), epidermal inclusion cysts (25\%), Bartholin duct cysts (27.5\%), and remaining $17.5 \%$ is constituted by Gartner duct cyst, endometriotic cysts and unclassified type. ${ }^{1,3,4}$ Gartner duct cysts represent embryologic remnants of the caudal end of the mesonephric (Wolffian) duct. These cysts are usually small and

\footnotetext{
${ }^{1,2}$ Senior Resident, ${ }^{3}$ Assistant Professor

${ }^{1-3}$ Department of Obstetrics and Gynecology, ESI Post Graduate Institute of Medical Sciences and Research Bengaluru, Karnataka, India
}

Corresponding Author: GS Anitha, Senior, Resident Department of Obstetrics and Gynecology, ESI Post Graduate Institute of Medical Sciences and Research, Bengaluru Karnataka, India, Phone: 08025711155, e-mail: aninaik85@ gmail.com asymptomatic and have been reported to occur in as many as $1 \%$ of all women. Because the ureteral bud also develops from the Wolffian duct, it is not surprising that Gartner duct cysts have been associated with ureteral and renal abnormalities, including congenital ipsilateral renal dysgenesis or agenesis, crossed fused renal ectopia and ectopic ureters. In addition, associated anomalies of the female genital tract, including structural uterine anomalies (ipsilateral müllerian duct obstruction, bicornuate uteri, and uterus didelphys) and diverticulosis of the fallopian tubes, have been described. Gartner cysts can be asymptomatic or can present with symptoms like mass per vagina, vaginal discharge, pain, dyspareunia, voiding problems, etc. ${ }^{1}$ Patients who present in adult life typically complain of dyspareunia, disfigurement, mass filling or fullness in the vagina. If discovered early in life, usually presents as an external genitalia mass, abdominal pain, vaginal discharge, recurrent urinary tract infection, incontinence, or enuresis. This report describes an unusual presentation of large anterior vaginal wall Gartner cyst.

\section{CASE REPORT}

A 17 years old unmarried girl came to our OPD with complaints of mass per vagina since one and a half year. Her complaints were insidious in onset and have gradually progressed. There is no history of associated bowel or bladder disturbances. There is no history of increase in size of the swelling on straining or lifting heavy weights. General and other systemic examinations were normal.

On local examination, external genitalia was normal. A $8 \times 4 \times 3 \mathrm{~cm}$ tense, nontender cystic swelling covered by the vaginal mucosa was seen in the anterior vaginal wall. Speculum examination revealed the swelling to be arising from the anterior vaginal wall. There was no impulse on cough, overlying vaginal rugosities were absent. Upper limit of the cyst was extending up to $1 \mathrm{~cm}$ below the anterior lip of the cervix and the lower limit was extending up to the urethral meatus. Bladder was catheterized and the cyst size was the same. On per rectal examination, uterus feels normal sized, nontender, mobile with both fornices free (Figs 1 and 2).

On investigations, blood biochemistry was essentially normal. Transvaginal ultrasonography showed a normal sized uterus and ovaries with endometrial thickness of $5.5 \mathrm{~mm}$. A well-defined uniloculated cystic swelling was seen located anterior to the cervix in the anterior vaginal 


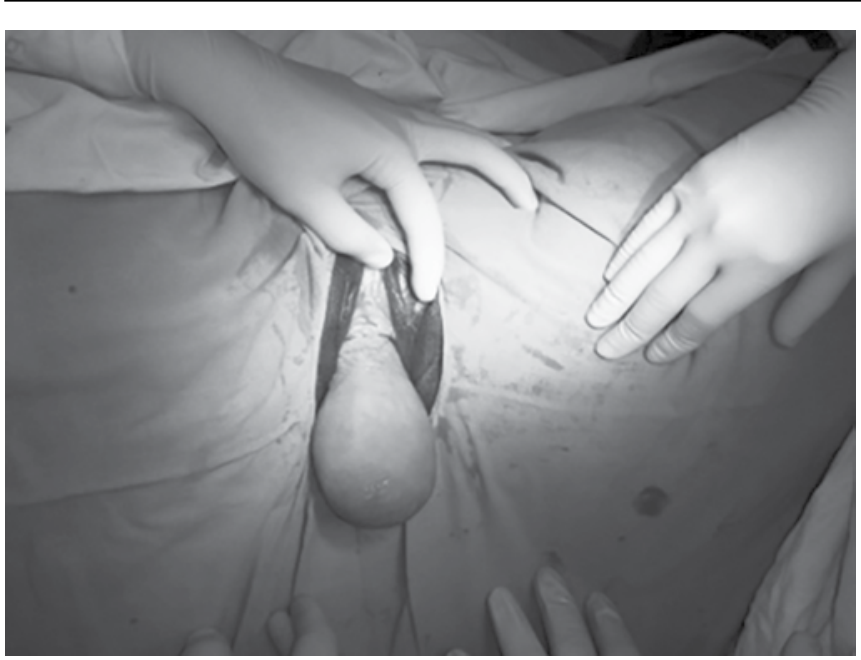

Fig. 1: Vaginal cyst

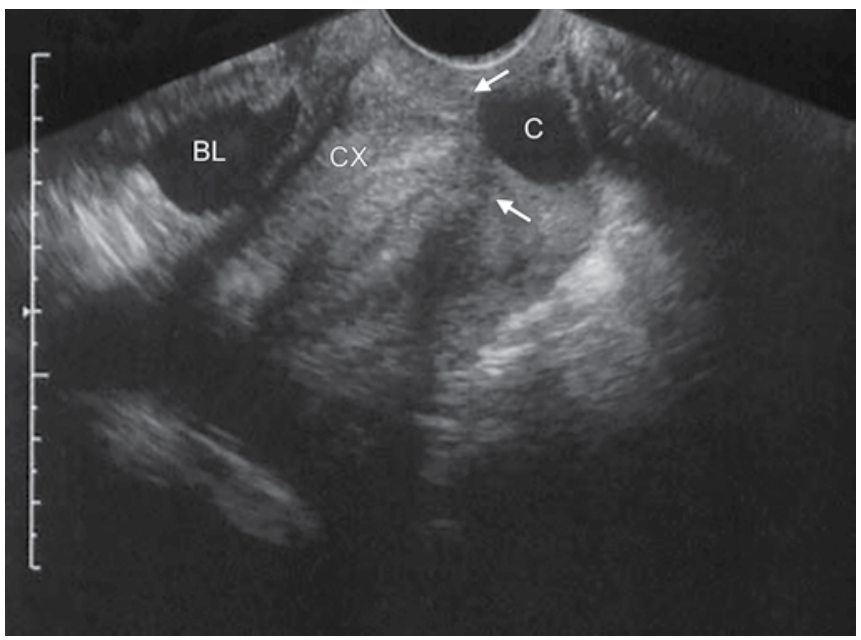

Fig. 3: Sagittal transvaginal ultrasonographic view of the pelvis. Gartner duct cyst $(C)$, and the clear anechoic line depicting the uterine cervix as separate from the cystic lesion (arrows) (BL: Bladder and CX: Cervix)

wall measuring $8 \times 4 \times 3 \mathrm{~cm}$ and volume of around $40 \mathrm{cc}$. There was thick content within the cyst and no evidence of fluid levels, calcifications or layering. No vascularity was seen within it. Transverse transvaginal scanning confirmed these findings and depicted the cyst lateral to the uterine cervix. Both kidneys were depicted ultrasonographically and appeared normal (Fig. 3).

\section{DIFFERENTIAL DIAGNOSIS}

Cystocele was excluded by catheterization. Endometriotic cyst was ruled out due to absence of pain. The location of the cyst ruled out Bartholin's cyst, which is located on the posterolateral vaginal wall. Gartner cyst and müllerian cyst are both usually located on the anterior or anterolateral aspect of the vaginal mucosa and the diagnosis is made by histopathological examination. Inclusion cysts of the vagina are small cysts found at the lower end of vagina on the posterior surface, arising from inclusion beneath the surface of tags of mucosa resulting from

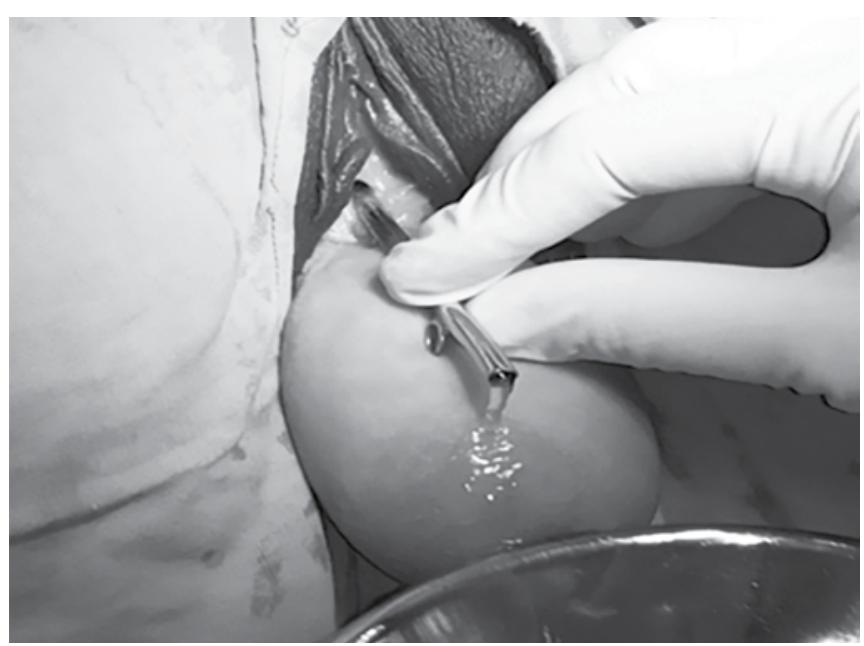

Fig. 2: Urethra is catheterized to show the anterior location of the cyst

perineal lacerations or from imperfect approximation in the course of surgical repair of the perineum. Histopathology of the lining epithelium of the cyst wall will differentiate inclusion cyst from Gartner cyst. Another rare differential diagnosis is specific obstructed Müllerian duct anomalies (usually uterus didelphys with obstructed hemivagina) which are not true cystic lesions and usually contain echogenic contents (obstructed menstrual debris), and patients with these lesions commonly have cyclic symptoms (primary dysmenorrhea). In contrast, patients with Gartner duct cysts are usually asymptomatic. Gartner cyst is differentiated from ureterocele in patients with ipsilateral renal dysgenesis. The cystis retrovesical and bulges into the bladder wall. The cyst does not communicate with the bladder, bladder neck or urethra and does not obviously change in shape and size when intravesical pressure increases with bladder filling and voiding.

\section{TREATMENT}

Patient underwent surgical excision of the cyst under spinal anesthesia. A small vertical incision was made on the anterior vaginal wall. The cyst was excised by sharp and blunt dissection. Care was taken to prevent urethral and bladder injury. Cyst was attached to the vagina by fibrous attachment. Excess of vaginal tissue was excised and vaginal mucosa was closed with absorbable sutures (Figs $4 \mathrm{~A}$ to D).

Patient had an uneventful postoperative period and was discharged on third postoperative day. She came back for follow-up after 2 weeks and then after 3 months and was asymptomatic.

The gross finding of the specimen was smooth, gray white externally.

Cut section showed smooth inner wall and contained mucoid material. Histopathological evaluation of the specimen revealed cellular remnants composed of 


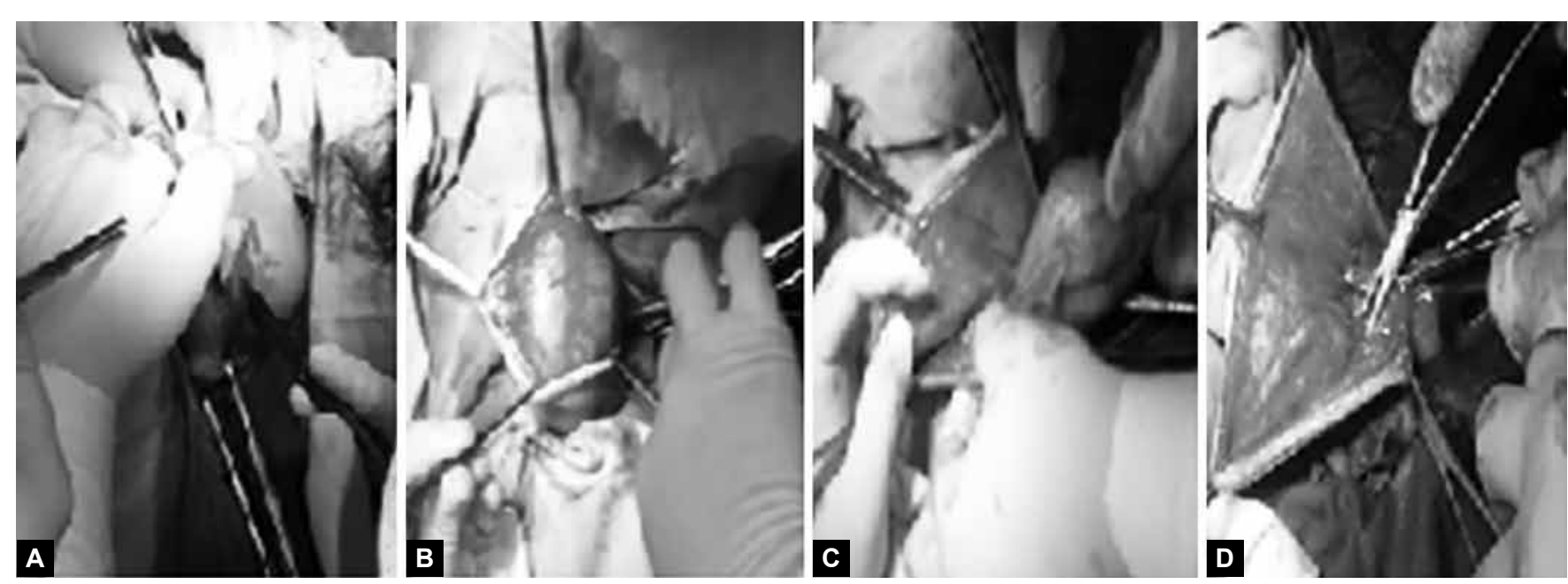

Figs 4A to D: (A) Anterior vertical incision, (B) separating the cyst wall, (C) enucleation of the cyst and (D) approximation of vaginal wall
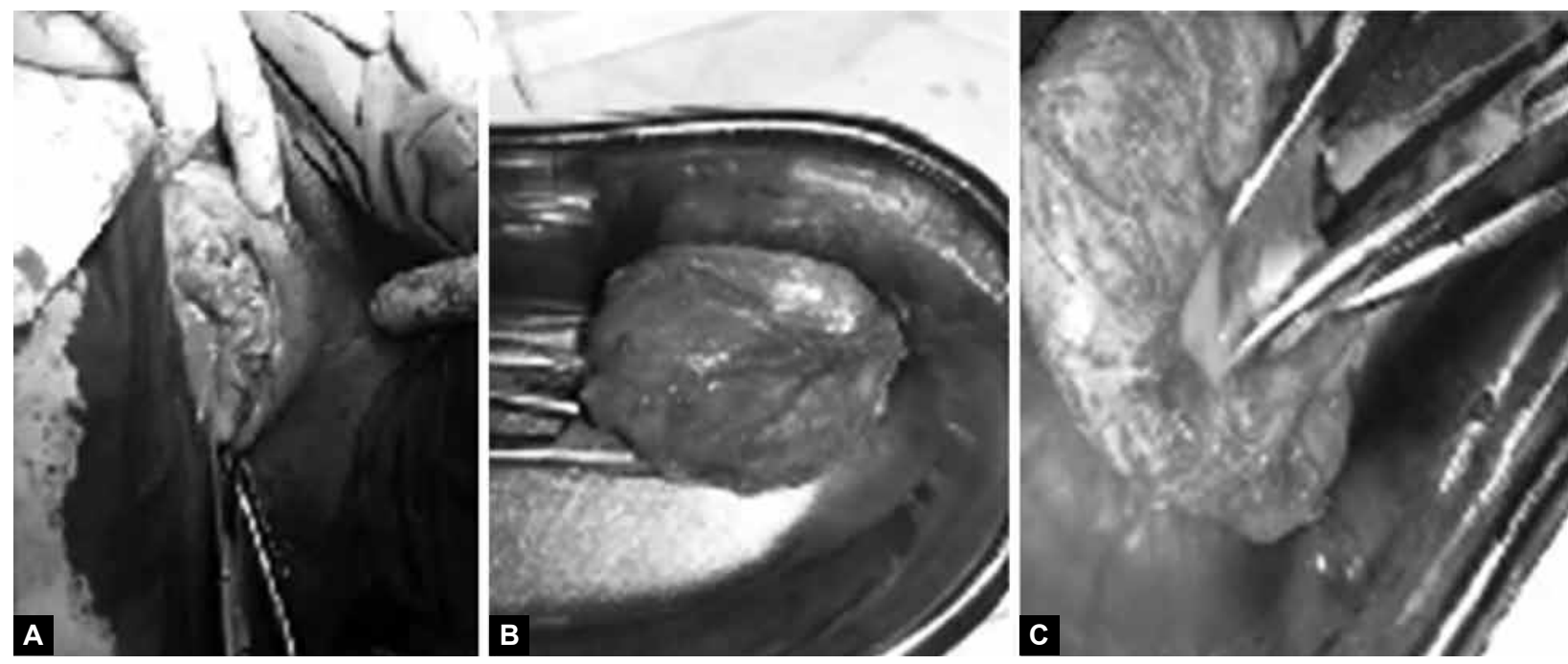

Figs 5A to C: (A) After closure, (B) excised cyst-gross and (C) mucoid material

non-mucin secreting low columnar or cuboidal epithelium. This was characteristic of Gartner cyst. The walls showed chronic inflammatory cell infiltrate and congestion (Figs 5A to C).

\section{DISCUSSION}

Vaginal cysts are reported in approximately 1 in 200 females. ${ }^{5}$ They are predominantly seen in women of reproductive age and also in children and postmenopausal women. ${ }^{6}$ During the 8 th week of embryologic development, the paired Müllerian (paramesonephric) ducts fuse distally and develop into the uterus, cervix and upper vagina, which are lined by a pseudostratified columnar (glandular) epithelium. Wolffian (mesonephric) ducts normally regress in the female, and their remnants include Gartner duct, epoophoron and paroophoron. Beginning at week 12 of intrauterine development, a squamous epithelial plate derived from the urogenital sinus begins to grow upward and replace the original pseudostratified columnar epithelium with squamous mucosa. ${ }^{7}$ The Gartner duct is the remnant of the vaginal portion of the Wolffian duct. Secretion by persistent glandular epithelium causes cystic dilatation, giving rise to Gartner duct cysts. True Gartner duct cysts are usually located along the anterolateral wall of the proximal third of the vagina. Gartner duct cysts can also be associated with abnormalities of the metanephric urinary system. Although such abnormalities usually present in childhood, awareness of this association should prompt the clinician to image the urinary tract in these patients. Ectopic ureters, besides having direct communications with the vagina and introitus, have been reported to communicate with Gartner duct cysts and cause urinary incontinence. ${ }^{8}$ The Gartner duct cyst may also represent an ipsilateral blind vagina, thus lending support to the hypothesis that the distal segment of the Wolffian duct contributes to the formation of the vagina.

Magnetic resonance imaging (MRI) is the imaging modality of choice for characterizing the cyst. ${ }^{7}$ These cysts have been observed in 1 to $2 \%$ of female pelvic MRI examinations. ${ }^{9}$ They are typically located in the anterolateral aspect of the proximal third of the vagina. They 
exhibit low-signal intensity on T1-weighted images and high signal intensity on T2-weighted images when they are simple cysts. When there is intracystic protein, mucin, or hemorrhage, they exhibit intermediate to high signal intensity on T1-weighted images. Neither the cyst nor its wall enhances after intravenous contrast injection. ${ }^{9}$ Most of these lesions are confined to the vaginal walls, but the larger cysts can extend into the ischiorectal fossa. Imaging modalities like transvaginal sonography and MRI are helpful in exact localization, number and communication with surrounding structures. ${ }^{10}$ Confirmation is by histopathological examination revealing cellular remnants composed of non-mucin secreting low columnar or cuboidal epithelium. The differential diagnoses of a cyst in the lateral aspects of the female genital tract include ovarian cysts, broad ligament cysts, nabothian cysts, Bartholin cysts, Gartner duct cyst, inclusion cysts and endometriotic cyst. Treatment is surgical excision of the vaginal cyst which must be done carefully to avoid injury to urethra and bladder. If surgical treatment is indicated, marsupialization or simple transvaginal excision is usually adequate. ${ }^{11}$ Surgical excision is frequently associated with bleeding due to the natural vascularity of vagina and vulva. This also might be associated with injury to the nearby structures, such as the urethra, bladder, and ureters, with subsequent genitourinary fistula development. In clitoral and hymenal Gartner cysts, injuries to the sensory nerves of erogenous areas as well as removal of much skin may result in sexual frigidity. Other postoperative complications include pain, hemorrhage and dyspareunia. Laser surgery can help in minimizing such complications. Abd-Rabbo and Atta described a novel method for management of such cysts with aspiration and $5 \%$ tetracycline sclerotherapy with good results and no side effects. ${ }^{11}$
Cyst marsupialization is a simple, minimally invasive procedure, created minimal surgical scarring. Long-term follow-up after such procedure proves its efficacy, with no demonstrated side effects or recurrence. ${ }^{12}$

Our case is of unusual and rare large anterior vaginal wall Gartner cyst.

\section{REFERENCES}

1. Rashmi, Suneja A, Agarwal N, et al. Vaginal Mullerian cyst presenting as enterocele. J Obstet Gynecol India 2009 JanFeb;59(1):74-76.

2. Rivlin ME, Meek GR, et al. Intracystic hemorrhage in a nonendometriotic Müllerian vaginal cyst. World J Clin Cases 2013 April 16;1(1):34-36.

3. Sahnidt WN. Pathology of the vagina-vaginal cysts. In: Fox H, Wella M, editors. Haines and Taylor Obstetrical and Gynecological pathology. Vol. 1, Fifth edition. New York, NY: Churchill Livingstone; 2003. p. 180-183.

4. Kondi-Pafiti A, Grapsa D, Papakonstatantinou K, et al. Vaginal cysts: a common pathological entity revisited. Clin Exp Obstet Gynecol 2008;35(1):41-44.

5. Hoffman, Schaffer, Schorge. Congenital vaginal cysts. In: Hwang, editor. Williams gynecology. 2nd ed. New York: Mc Graw Hill Medical; 2009. p. 495.

6. Junaid TA, Thomas SM. Cysts of vulva and vagina: a comparative study. Int J Gynecol Obstet 1981;19:239-243.

7. Eilber KS, Raz S. Benign cystic lesions of the vagina: a literature review. J Urol 2003;170(3):717-722.

8. Kier R. Non ovarian gynecologic cysts: MR findings. Am J Roentgenol 1992;158(6):1265-1269.

9. Hagspiel KD. Giant Gartner duct cyst: magnetic resonance imaging findings. Abdom Imaging 1995;20(6):566-568.

10. Pradhan J, Tobon H. Vaginal cysts: a clinicopathological study of 41 cases. Int J Gynecol Pathol 1986;5(1):35-46.

11. Abd-Rabbo MS, Atta MA. Aspiration and tetracycline sclerotherapy: a novel method for management of vaginal and vulval Gartner cysts. Int J Gynaecol Obstet 1991 Jul;35(3): 235-237.

12. Binsaleh S, Al-Assiri M, Jednak R, El-Sherbiny M. Gartner duct cyst simplified treatment approach. Int Urol Nephrol 2007;39(2):485-487. 